\title{
Kidney Function and Risk of Physical and Cognitive Impairment in Older Persons with Type 2 Diabetes at an Outpatient Clinic with Geriatric Assessment Implementation
}

\author{
Cheng-Fu Lin ${ }^{1,2}$ \\ Hsiu-Chen $\mathrm{Liu}^{3}$ \\ Shih-Yi Lin ${ }^{1,4,5}$ \\ 'Center for Geriatrics \& Gerontology, \\ Taichung Veterans General Hospital, \\ Taichung, 40705, Taiwan; ${ }^{2}$ Division of \\ Occupational Medicine, Department of \\ Emergency, Taichung Veterans General \\ Hospital, Taichung, 40705, Taiwan; \\ ${ }^{3}$ Department of Nursing, Taichung \\ Veterans General Hospital, Taichung, \\ 40705, Taiwan; ${ }^{4}$ School of Medicine, \\ National Yang Ming Chiao Tung \\ University, Taipei, II22I, Taiwan; \\ ${ }^{5}$ Institute of Clinical Medicine, School of \\ Medicine, National Yang Ming Chiao Tung \\ University, Taipei, II22I, Taiwan
}

Purpose: Diabetes is associated with an increased risk of cognitive and physical functional decline that may impede disease self-management. By incorporating cognitive and physical function assessment, this study aimed to evaluate prevalence and factors associated with cognitive and physical dysfunction in older diabetic people.

Methods: The cross-sectional study was performed from August 1, 2017 to November 30, 2018. The patients aged 65 years or older with type 2 diabetes mellitus were enrolled and the disease was routinely evaluated by blood hemoglobin A1c (A1C), blood pressure, lipids, and kidney function measured by estimated glomerular filtration rate (eGFR) and urinary albumin-creatinine rate (UACR). Besides, cognitive dysfunction through Mini-mental State Examination (MMSE), and functional disabilities by Activities of Daily Living (ADL) questionnaire were assessed simultaneously.

Results: Among 863 patients $(48.3 \%$ men) with a median age of 72.0 years (interquartile range or IQR: $67.0-78.0$ years), 159 (18.5\%) had cognitive impairment assessed by MMSE, while $40(4.6 \%)$ experienced at least one problem in ADL. With different A1C stratifications, it was shown that both MMSE and ADL scores were associated with glycemic control. Patients with impaired MMSE and ADL scores were older, had lower eGFR, lower blood pressure, and higher UACR levels. After adjustment of possible confounders, it was shown that age and eGFR predicted MMSE and ADL score impairment.

Conclusion: By incorporating physical and cognitive function screening program into routine care at a diabetes outpatient clinic, our study found that both cognitive and physical function impairment were common in older diabetic patients, and their relevant factors, including older age, and lower eGFR. It was recommended that in older individuals with diabetes, particularly those with risk factors, an additional assessment of cognitive and physical functions can be integrated into routine clinical process to provide more comprehensive management plans.

Keywords: diabetes, aged, outpatient, functional decline, cognitive impairment

\section{Introduction}

Diabetes is a risk factor for cognitive impairment and dementia. Individuals diagnosed with diabetes are 1.5 times more likely to experience cognitive decline and early stage of dementia than individuals without diabetes. ${ }^{1}$ The abnormalities in cognitive functions due to dementia may cause significant difficulty in the management of the disease, and may also impede self-management behavior, particularly given the high complexity of diabetes treatment regimens (eg, blood glucose
Correspondence: Shih-Yi Lin

Center for Geriatrics \& Gerontology, Taichung Veterans General Hospital, 1650 Taiwan Boulevard Sect. 4, Taichung, 40705, Taiwan

Tel +886-4-2359-2525\#3390

Fax +886-4-2359-5046

Email sylin@vghtc.gov.tw 
testing, meal planning and medication adhesion). ${ }^{2}$ Importantly, patients experiencing such cognitive disorders are unlikely to self-report either their cognitive problems or difficulty in managing their diabetes, requiring early identification of cognitive disorder in diabetic patients by health-care providers be imperative.

In addition to increasing the risk of cognitive dysfunction, epidemiological studies have indicated that individuals with diabetes generally have a 2 to 3 times greater risk of functional disability in terms of Activities of Daily Living (ADL), Instrumental Activities of Daily Living (IADL), and mobility-related tasks when compared with individuals without diabetes. ${ }^{3}$ The physical functional decline seen among diabetic patients is associated with disease control and its complications. ${ }^{3}$ As a consequence, patients were at a high risk for more advanced dependence, hospitalizations, and a greater need for disabilityrelated health resources.

Previous studies performed in Taiwan have reported an increased risk of physical disability, cognitive impairment, and depression symptoms in diabetic patients. ${ }^{4-6}$ However, the association between diabetic complications, metabolic controls and prescribed medications with physical and cognitive disability have been less detailed. In Taiwan's diabetes pay-for-performance program, vascular complications, blood glucose, lipid, and blood pressure control examinations as well as diabetes education are performed regularly at diabetes outpatient clinics. ${ }^{7}$ It has been hypothesized that through additional cognition and physical function assessment their association with glycemic control and other diabetes-relevant factors in older adults with diabetes can be elucidated, and benefit for management and support in these population. In this study, we aimed to incorporate assessment of cognitive and physical functions at a diabetes outpatient clinic, and examine the prevalence of physical, cognitive impairment and explore potential risk factors in older adults with diabetes.

\section{Materials and Methods Study Design}

Since August 1, 2017, our diabetes outpatient clinics have incorporated the Mini-mental State Examination (MMSE), 5-item Geriatric Depression Scale (GDS-5), ADL and IADL scale assessments into the diabetes pay-forperformance program in Taiwan. At the annual visit of diabetes pay-for-performance program, evaluation of diabetic complications, blood glucose, lipid, blood pressure control, and other laboratory examinations were all required as well as diabetes education. ${ }^{7}$ When diabetes education was performed, cognitive and physical functions were also assessed by well-trained nurse educators. In the study, the baseline survey data collected between August 1, 2017 and November 30, 2018 were analyzed anonymously in cross-sectional manner. The study was approved by the Institutional Review Board (IRB) of the hospital (No: CE18117A), with the IRB waiving the requirement of verbal or written consent from the enrolled subjects and the patient data accessed was treated with confidentiality.

\section{Participants}

Subjects who were aged 65 years or older with a diagnosis of type 2 diabetes mellitus, and had participated in the diabetes pay-for-performance program at our diabetes outpatient clinic were enrolled in the study. Participants with an established diagnosis of dementia, those needing an interpreter, or those with severe visual impairment (unable to complete the tests) were excluded. Based upon previous reports, ${ }^{1-3}$ we estimated that there would be a prevalence of $10 \%$ of the subjects experiencing cognitive impairment and $40 \%$ having physical function impairment in older persons with diabetes. We used $5 \%$ as a margin error, and $95 \%$ as the confidence interval in order to calculate the sample size. Accordingly, the minimal sample size should be no less than 369. Ultimately, a total of 863 patients were enrolled between August 1, 2017 and November 30, 2018.

\section{Demographics and Clinical Information of Participants}

Medical records of all enrolled participants were reviewed, including age, gender, education level, comorbidities, medications, disease duration, Body Mass Index (BMI), blood pressure, and laboratory examinations, including hemoglobin A1c (A1C) measured using a boronate affinity high-performance liquid chromatography method (Trinity Biotech Premier Hb9210, Ireland), fasting plasma glucose (FPG), triglyceride (TG), low-density lipoproteins (LDL) cholesterol, creatinine $(\mathrm{Cr})$, and urine albumin-creatinine ratio (UACR) with a chemistry analyzer (Hitachi Labospect 008, Tokyo, Japan), and estimated glomerular filtration rate (eGFR) determined by the formula of the Modification of Diet in Renal Disease (MDRD) equation for Taiwanese adults. ${ }^{8}$ 


\section{Measurement of Cognitive Function}

The MMSE for cognitive screening included specific questions related to attention, orientation, memory, calculation, and language. The score range was from 0 to 30 and in accordance with the patient's educational level, with the stratification of the cut-off points for possible dementia determined as follows: MMSE score 24 if the patient was literate; MMSE score 13 if the patient was illiterate. ${ }^{9}$ The GDS-5 was used for depressive symptoms where a score of 2 or higher indicated depressive symptoms. ${ }^{10}$

\section{Measurement of Physical Function}

Results from both the Barthel index for ADL and the Lawton IADL scale were evaluated for activities of daily living, with most of the patients completing the questionnaires on their own. The Barthel index of ADL assesses the basic ability to meet one's own physical needs, including feeding, ambulating, dressing, personal hygiene, continence, and toileting, and is scaled between 0 and 100 with low scores representing the severity of dependence. ${ }^{8}$ The ADL cutoff scores were defined as $\mathrm{ADL}=100$ for an independent and $\mathrm{ADL}<100$ for one who is non-independent. ${ }^{8}$ The Lawton IADL scale assesses more complex skills, including the ability to properly use transportation, shopping, managing of finances, taking medications, meal preparation, housecleaning, and communication with others, and is scaled between 0 and 8 , with low scores indicating that the patient requires outside assistance. $^{8}$

\section{Determination of Potential Risk Factors}

Potential risk factors were examined included clinical information, demographics, and geriatric assessment of cognitive and physical functions. All assessments were performed by a well-trained nurse educator and the variables listed were potential confounders, therefore, warranted inclusion in the statistical models.

\section{Statistical Analysis}

Continuous variables were expressed as median and interquartile range (IQR, 25\%-75\%). Categorical data were expressed as number and percentage. Comparisons between quantitative groups were performed using the MannWhitney $U$-test, while the chi-square test/Fisher's exact test were used for categorical ones. Univariate and multivariable logistic regression models were used to examine the associations between binary outcomes (normal and impaired MMSE, ADL, and IADL scores) and covariates (age, gender, disease duration, comorbidities, diabetic complications, medications, and physical and laboratory examination data). Due to the exploratory nature of the analysis for the outcomes in this study, no correction for multiple comparisons was performed. ${ }^{11,12}$ Statistical analyses were performed using SPSS version 22.0 (SPSS Inc., Chicago, IL, USA). Statistical significance was set at $\mathrm{p}<0.05$.

\section{Results \\ Baseline Characteristics of the Participants}

The characteristics of the study population and the treatment modalities are shown in Table 1. The median age was 72.0 years (interquartile range or IQR: $67.0-78.0$ years) with a female-to-male ratio of 52:48. The educational levels were: $13.4 \%$ illiterate, and $86.6 \%$ literate. Insulin (not including oral insulin medication) was used by $4.8 \%$ of the patients. The most common comorbidities were hyperlipidemia, hypertension, and nephropathy. Polypharmacy (currently using $>4$ drugs) was occurring in 512 patients. In total, $159(18.5 \%)$ of all patients had an MMSE score below the cutoff value, while $8.3 \%$ of the patients had a GDS score greater or equal to 2. Forty (4.6\%) patients had at least one physical functional problem assessed by ADL, while 470 (54.5\%) patients had at least one abnormal IADL component. The median A1C, representing glycemic control, was 7.1 (IQR: 6.5-7.9).

\section{Comparison According to AIC}

According to different A1C stratifications, good glycemic control is defined as an A1C level $<7.0 \%$, intermediate glycemic control an A1C level of 7.0-9.0\%, and poor glycemic control an $\mathrm{A} 1 \mathrm{C}$ level $\geq 9.0 \%$. It was shown that diabetes duration, educational level, polypharmacy, BMI, FPG, TG, UACR, MMSE, and ADL scores were all associated with glycemic control status (Table 2). However, impaired ADL, IADL, and MMSE scores were not associated with other diabetic complications, including cardiovascular disease, neuropathy and retinopathy (Tables 2-5).

\section{The Association Factors of Cognitive Impairment}

Patients with impaired MMSE scores were older, had higher blood $\mathrm{Cr}$, lower eGFR, lower diastolic blood pressure, and greater UACR levels (Table 3). Using univariate regression analysis, it was shown that age, diastolic blood pressure, presence of cerebral vascular disease, disease duration, A1C, FPG, Cr, eGFR, ADL, IADL and GDS-5 
Table I Baseline Characteristics of the Participants

\begin{tabular}{|c|c|c|}
\hline Demographic Characteristics & & \\
\hline Age (years) & 72.0 & $(67.0-78.0)$ \\
\hline \multicolumn{3}{|l|}{ Sex, n(\%) } \\
\hline Female & 446 & $(51.7 \%)$ \\
\hline Male & 417 & $(48.3 \%)$ \\
\hline \multicolumn{3}{|l|}{ Educational level, $n(\%)$} \\
\hline Illiterate & 115 & $(13.4 \%)$ \\
\hline Literate & 745 & $(86.6 \%)$ \\
\hline \multicolumn{3}{|l|}{ Geriatric characteristics } \\
\hline \multicolumn{3}{|l|}{ Polypharmacy, n (\%) } \\
\hline No & 351 & $(40.6 \%)$ \\
\hline Yes & 512 & $(59.3 \%)$ \\
\hline Mini-mental state examination & 27.0 & $(24.0-29.0)$ \\
\hline 5-item Geriatric Depression Scale & 0 & $(0-1.0)$ \\
\hline Barthel index & 100.0 & $(100.0-100.0)$ \\
\hline Lawton scale & 7.0 & $(6.0-8.0)$ \\
\hline \multicolumn{3}{|l|}{ Diabetes characteristics } \\
\hline Duration (years) & 13.0 & $(7.0-19.0)$ \\
\hline Cardiovascular disease & 572 & $(66.3 \%)$ \\
\hline Neuropathy & 82 & $(9.5 \%)$ \\
\hline Retinopathy & 27 & $(3.1 \%)$ \\
\hline Body mass index $\left(\mathrm{kg} / \mathrm{m}^{2}\right)$ & 24.8 & $(22.5-27.3)$ \\
\hline Systolic blood pressure $(\mathrm{mmHg})$ & 130.0 & $(121.0-140.0)$ \\
\hline Diastolic blood pressure $(\mathrm{mmHg})$ & 72.0 & $(63.0-79.0)$ \\
\hline Blood hemoglobin Alc (\%) & 7.1 & $(6.5-7.9)$ \\
\hline Fasting plasma glucose (mg/dL) & 130.0 & $(|1| 2.0-153.0)$ \\
\hline Triglycerides (mg/dL) & 104.0 & $(73.0-150.0)$ \\
\hline Low-density lipoprotein cholesterols (mg/dL) & 82.0 & $(68.0-99.0)$ \\
\hline Blood creatinine (mg/dL) & 1.0 & $(0.8-1.3)$ \\
\hline Estimated glomerular filtration rate $\left(\mathrm{mL} / \mathrm{min} / 1.73 \mathrm{~m}^{2}\right)$ & 70.4 & $(50.9-87.0)$ \\
\hline Urine albumin-creatinine ratio $(\mathrm{mg} / \mathrm{g})$ & 25.2 & $(9.6-99.7)$ \\
\hline \multicolumn{3}{|l|}{ Treatment } \\
\hline Lifestyle modification & 87 & $(10.1 \%)$ \\
\hline Oral medications only & 593 & $(68.7 \%)$ \\
\hline Insulin only & 41 & $(4.8 \%)$ \\
\hline Oral medications + insulin & 142 & $(16.5 \%)$ \\
\hline
\end{tabular}

were all associated with an increased odds ratio of impaired MMSE. When these variables were further put into multivariate logistic regression analyses, age, presence of cerebral vascular disease, and eGFR were still significantly associated with an increased odds ratio of impaired MMSE (Table 6).

\section{The Association Factors of Physical Dysfunction}

Those patients with an ADL $<100$ were also older, had higher blood $\mathrm{Cr}$, lower eGFR, lower systolic blood pressure and greater UACR values (Table 4). Regarding impaired ADL, it was shown that age, gender, systolic blood pressure, eGFR, IADL, GDS-5 and MMSE were all positively associated with an increased odds ratio of abnormal ADL in univariate analysis. After adjustment, we found that age, gender, systolic blood pressure and eGFR remained positively correlated with ADL abnormality (Table 6). Patients with an IADL $<8$ were predominantly male, at an older age, had higher blood $\mathrm{Cr}$, lower eGFR and greater UACR values (Table 5). With respect to IADL, it was found that age, gender, presence of nephropathy, disease duration, diabetes treatment, Cr, eGFR, MMSE and ADL were all positively associated with an increased odds ratio of abnormal IADL in univariate analysis. After adjustment, we found that age, gender and 
Table 2 Characteristics of Participants According to AIC

\begin{tabular}{|c|c|c|c|c|c|c|c|}
\hline \multirow{4}{*}{$\begin{array}{l}\text { Characteristics } \\
\text { Demographic characteristics } \\
\text { Age }\end{array}$} & \multicolumn{6}{|c|}{ AIC } & \multirow[t]{2}{*}{$p$ value } \\
\hline & \multicolumn{2}{|c|}{$<7(n=37 I)$} & \multicolumn{2}{|c|}{$7 \leq$ Value $<9(n=405)$} & \multicolumn{2}{|c|}{$\geq 9(n=87)$} & \\
\hline & & & & & & & \\
\hline & 73.0 & $(68.0-78.0)$ & 71.0 & $(67.0-77.0)$ & 72.0 & $(69.0-79.0)$ & 0.068 \\
\hline Gender, n(\%) & & & & & & & 0.107 \\
\hline Female & 177 & $(47.7 \%)$ & 224 & $(55.3 \%)$ & 45 & (51.7\%) & \\
\hline Male & 194 & $(52.3 \%)$ & 181 & $(44.7 \%)$ & 42 & $(48.3 \%)$ & \\
\hline Educational level, $n(\%)$ & & & & & & & 0.540 \\
\hline Illiterate & 54 & $(14.6 \%)$ & 52 & $(12.8 \%)$ & 9 & $(10.5 \%)$ & \\
\hline Literate & 315 & $(85.4 \%)$ & 353 & $(87.2 \%)$ & 77 & (89.5\%) & \\
\hline \multicolumn{8}{|l|}{ Geriatric characteristics } \\
\hline Polypharmacy, n(\%) & & & & & & & $<0.001$ \\
\hline No & 188 & $(50.7 \%)$ & 142 & $(35.1 \%)$ & 21 & $(24.1 \%)$ & \\
\hline Yes & 183 & $(49.3 \%)$ & 263 & $(64.9 \%)$ & 66 & $(75.9 \%)$ & \\
\hline Mini-mental state examination & 27.0 & $(24.0-29.0)$ & 27.0 & $(24.0-29.0)$ & 26.0 & $(22.0-28.0)$ & 0.027 \\
\hline 5-item Geriatric Depression Scale & 0 & $(0-1.0)$ & 0 & $(0-1.0)$ & 0 & $(0-1.0)$ & 0.679 \\
\hline Barthel index & 100.0 & $(100.0-100.0)$ & 100.0 & $(100.0-100.0)$ & 100.0 & $(100.0-100.0)$ & 0.014 \\
\hline Lawton scale & 7.0 & $(6.0-8.0)$ & 7.0 & $(6.0-8.0)$ & 7.0 & $(6.0-8.0)$ & 0.161 \\
\hline \multicolumn{8}{|l|}{ Diabetes characteristics } \\
\hline Duration (years) & 11.0 & $(6.0-16.0)$ & 15.0 & $(9.0-21.0)$ & 16.0 & $(11.0-22.0)$ & $<0.001$ \\
\hline Cardiovascular disease, $\mathrm{n}(\%)$ & & & & & & & 0.214 \\
\hline No & 129 & $(34.8 \%)$ & 140 & $(34.6 \%)$ & 22 & $(25.3 \%)$ & \\
\hline Yes & 242 & $(65.2 \%)$ & 265 & $(65.4 \%)$ & 65 & $(74.7 \%)$ & \\
\hline Neuropathy, n(\%) & & & & & & & 0.028 \\
\hline No & 346 & (93.3\%) & 361 & (89.1\%) & 74 & $(85.1 \%)$ & \\
\hline Yes & 25 & $(6.7 \%)$ & 44 & $(10.9 \%)$ & 13 & $(14.9 \%)$ & \\
\hline Retinopathy, n(\%) & & & & & & & 0.426 \\
\hline No & 362 & $(97.6 \%)$ & 389 & (96.1\%) & 85 & (97.7\%) & \\
\hline Yes & 9 & $(2.4 \%)$ & 16 & $(3.9 \%)$ & 2 & $(2.3 \%)$ & \\
\hline Body mass index $\left(\mathrm{kg} / \mathrm{m}^{2}\right)$ & 24.7 & $(22.3-27.1)$ & 24.8 & $(22.7-27.3)$ & 25.4 & $(23.3-29.6)$ & 0.040 \\
\hline Systolic blood pressure $(\mathrm{mmHg})$ & 130.0 & $(\mid 20.0-138.0)$ & 130.0 & $(|22.0-| 40.5)$ & 130.0 & $(119.0-143.0)$ & 0.130 \\
\hline Diastolic blood pressure $(\mathrm{mmHg})$ & 71.0 & $(63.0-78.0)$ & 73.0 & $(65.0-80.0)$ & 69.0 & $(59.0-80.0)$ & 0.200 \\
\hline Fasting plasma glucose (mg/dL) & 120.0 & $(107.0-133.0)$ & 138.0 & $(119.0-163.5)$ & 184.0 & $(|30.0-2| 3.0)$ & $<0.001$ \\
\hline Triglycerides (mg/dL) & 98.0 & $(69.0-139.0)$ & 108.0 & $(76.0-159.0)$ & 131.0 & $(76.0-184.0)$ & $<0.001$ \\
\hline Low-density lipoprotein cholesterols (mg/dL) & 82.0 & $(68.0-95.0)$ & 82.0 & $(68.0-101.0)$ & 82.0 & $(63.0-106.0)$ & 0.323 \\
\hline Blood creatinine (mg/dL) & 1.0 & $(0.8-1.3)$ & 1.0 & $(0.8-1.3)$ & 1.0 & $(0.7-1.4)$ & 0.227 \\
\hline Estimated glomerular filtration rate $\left(\mathrm{mL} / \mathrm{min} / 1.73 \mathrm{~m}^{2}\right)$ & 70.1 & $(50.2-84.8)$ & 71.5 & $(53.0-87.9)$ & 68.1 & $(45.2-88.7)$ & 0.489 \\
\hline Urine albumin-creatinine ratio $(\mathrm{mg} / \mathrm{g})$ & 19.2 & $(8.1-67.1)$ & 28.2 & $(10.0-119.3)$ & 43.9 & $(16.8-467.1)$ & $<0.001$ \\
\hline Treatment & & & & & & & $<0.001$ \\
\hline Lifestyle modification & 43 & $(11.6 \%)$ & 36 & $(8.9 \%)$ & 8 & $(9.2 \%)$ & \\
\hline Oral medications only & 291 & $(78.4 \%)$ & 264 & $(65.2 \%)$ & 38 & (43.7\%) & \\
\hline Insulin only & 10 & $(2.7 \%)$ & 24 & $(5.9 \%)$ & 7 & $(8.1 \%)$ & \\
\hline Oral medications + insulin & 27 & $(7.3 \%)$ & 81 & $(20.0 \%)$ & 34 & (39.1\%) & \\
\hline
\end{tabular}

diabetic treatment regimens remained positively associated with IADL abnormality (Table 6).

\section{Discussion}

In this study, cognitive and physical assessments conducted in a diabetes clinic revealed that $18.5 \%$ of patients had a low MMSE score, and 4.6\% and 54.5\% had at least one abnormal component in the ADL and IADL instruments, respectively. Moreover, age, renal function, blood pressure, and A1C control status were all associated with an increased risk of impaired MMSE and ADL scores. In line with previous studies, our study findings provide 
Table 3 Characteristics of Participants with and without Possible Dementia by MMSE ${ }^{\dagger}$

\begin{tabular}{|c|c|c|c|c|c|}
\hline \multirow{3}{*}{$\begin{array}{l}\text { Demographic characteristics } \\
\text { Age (years) }\end{array}$} & \multicolumn{2}{|c|}{ Normal } & \multicolumn{2}{|c|}{ Possible Dementia } & \multirow[t]{2}{*}{ p value } \\
\hline & & & & & \\
\hline & 71.0 & $(67.0-76.5)$ & 76.0 & $(71.0-83.0)$ & $<0.001$ \\
\hline Gender, n (\%) & & & & & 0.274 \\
\hline Female & 356 & $(50.8 \%)$ & 89 & $(56.0 \%)$ & \\
\hline Male & 345 & $(49.2 \%)$ & 70 & $(44.0 \%)$ & \\
\hline Educational level, n (\%) & & & & & $<0.001$ \\
\hline Illiterate & 112 & $(16.0 \%)$ & 3 & $(1.9 \%)$ & \\
\hline Literate & 589 & $(84.0 \%)$ & 156 & $(98.1 \%)$ & \\
\hline \multicolumn{6}{|l|}{ Geriatric characteristics } \\
\hline Polypharmacy & 406 & $(57.9 \%)$ & 104 & $(65.4 \%)$ & 0.100 \\
\hline Mini-mental state examination & 28.0 & $(26.0-29.0)$ & 22.0 & $(20.0-23.0)$ & $<0.001$ \\
\hline 5-item Geriatric Depression Scale & 0 & $(0-1.0)$ & 0 & $(0-1.0)$ & 0.003 \\
\hline Barthel index & 100.0 & $(100.0-100.0)$ & 100.0 & $(100.0-100.0)$ & $<0.001$ \\
\hline Lawton scale & 7.0 & $(6.0-8.0)$ & 6.0 & $(4.0-8.0)$ & $<0.001$ \\
\hline \multicolumn{6}{|l|}{ Diabetes characteristics } \\
\hline Duration (years) & 13.0 & $(7.0-19.0)$ & 16.0 & $(9.0-21.0)$ & 0.011 \\
\hline Cardiovascular disease & 465 & $(66.3 \%)$ & 105 & $(66.0 \%)$ & 1.000 \\
\hline Neuropathy & 62 & $(8.8 \%)$ & 20 & $(12.6 \%)$ & 0.194 \\
\hline Retinopathy & 25 & $(3.6 \%)$ & 2 & $(1.3 \%)$ & 0.204 \\
\hline Body mass index $\left(\mathrm{kg} / \mathrm{m}^{2}\right)$ & 24.8 & $(22.6-27.3)$ & 24.9 & $(22.4-27.5)$ & 0.632 \\
\hline Systolic blood pressure $(\mathrm{mmHg})$ & 130.0 & $(\mid 22.0-139.0)$ & 129.0 & $(118.0-145.0)$ & 0.888 \\
\hline Diastolic blood pressure $(\mathrm{mmHg})$ & 72.0 & $(64.0-79.0)$ & 70.0 & $(60.0-78.0)$ & 0.021 \\
\hline Blood hemoglobin Alc (\%) & 7.1 & $(6.5-7.8)$ & 7.2 & $(6.5-8.1)$ & 0.161 \\
\hline Fasting plasma glucose (mg/dL) & 129.0 & $(1 \mid 3.0-151.0)$ & 134.0 & $(110.0-167.0)$ & 0.288 \\
\hline Triglycerides (mg/dL) & 103.0 & $(73.0-147.5)$ & 104.0 & $(74.0-154.0)$ & 0.812 \\
\hline Low-density lipoprotein cholesterols $(\mathrm{mg} / \mathrm{dL})$ & 82.0 & $(68.0-98.0)$ & 84.0 & $(68.0-99.0)$ & 0.500 \\
\hline Blood creatinine (mg/dL) & 1.0 & $(0.8-1.3)$ & I.I & $(0.9-1.5)$ & $<0.001$ \\
\hline Estimated glomerular filtration rate $\left(\mathrm{mL} / \mathrm{min} / 1.73 \mathrm{~m}^{2}\right)$ & 71.9 & $(53.7-87.8)$ & 60.9 & $(41.6-78.6)$ & $<0.001$ \\
\hline Urine albumin-creatinine ratio $(\mathrm{mg} / \mathrm{g})$ & 21.3 & $(9.0-83.4)$ & 40.4 & $(\mid 3.6-180.7)$ & $<0.001$ \\
\hline Treatment & & & & & 0.027 \\
\hline Lifestyle modification & 71 & $(10.1 \%)$ & 16 & $(10.1 \%)$ & \\
\hline Oral medications only & 496 & $(70.8 \%)$ & 96 & $(60.4 \%)$ & \\
\hline Insulin only & 31 & $(4.4 \%)$ & 9 & $(5.7 \%)$ & \\
\hline Oral medications + insulin & 103 & $(14.7 \%)$ & 38 & $(23.9 \%)$ & \\
\hline
\end{tabular}

Notes: ${ }^{\dagger}$ Literate \& mini-mental state examination $\leq 24 /$ Illiterate \& mini-mental state examination $\leq 13$.

Abbreviation: MMSE, Mini-mental State Examination.

additional evidence surrounding the impact of diabetes on cognition and physical function. ${ }^{1-3}$ As cognitive and physical dysfunction have both been associated with a poorer ability in self-care and glycemic control for diabetics, additional assessments regarding cognition and physical functions in older persons with diabetes are recommended. ${ }^{13}$

There are several notable advantages of this study. First, the present study by combining physical and cognitive function assessment service and routine care in older diabetic patients showed these geriatric assessments can be used as one of the components towards more comprehensive care in diabetes. Other fields, such as oncology, nephrology, have previously reported studies on the pivotable role of geriatric assessment for treatment decisions and plans, ${ }^{14,15}$ while such initiatives were less evaluated in the field of diabetes care. Overall, this study addressed one of the priority issues specified by American Diabetes Association "Comprehensive Medical Evaluation and Assessment of Comorbidities" by developing a feasible geriatric assessment service for older diabetic patients to identify areas of vulnerability that might affect an older individual's ability to tolerate treatment and lead to rationale interventions to optimize diabetes care. ${ }^{16}$ 
Table 4 Characteristics of Participants with and without Dependence by $\mathrm{ADL}^{\dagger}$

\begin{tabular}{|c|c|c|c|c|c|}
\hline \multirow{3}{*}{$\begin{array}{l}\text { Demographic characteristics } \\
\text { Age (years) }\end{array}$} & \multicolumn{2}{|c|}{ ADL Independence } & \multicolumn{2}{|c|}{ ADL Dependence } & \multirow[t]{2}{*}{ p value } \\
\hline & & & & & \\
\hline & 72.0 & $(67.0-78.0)$ & 79.5 & $(73.3-86.0)$ & $<0.001$ \\
\hline Gender, n (\%) & & & & & 0.011 \\
\hline Female & 417 & $(50.7 \%)$ & 29 & $(72.5 \%)$ & \\
\hline Male & 406 & $(49.3 \%)$ & II & $(27.5 \%)$ & \\
\hline Educational level, n (\%) & & & & & 0.014 \\
\hline Illiterate & 104 & $(12.7 \%)$ & II & $(27.5 \%)$ & \\
\hline Literate & 716 & $(87.3 \%)$ & 29 & $(72.5 \%)$ & \\
\hline \multicolumn{6}{|l|}{ Geriatric characteristics } \\
\hline Polypharmacy & 484 & $(58.8 \%)$ & 28 & $(70.0 \%)$ & 0.214 \\
\hline Mini-mental state examination & 27.0 & $(24.0-29.0)$ & 20.5 & $(17.0-25.0)$ & $<0.001$ \\
\hline 5-item Geriatric Depression Scale & 0 & $(0-1.0)$ & 1.0 & $(0-1.0)$ & $<0.001$ \\
\hline Barthel index & 100.0 & $(100.0-100.0)$ & 80.0 & $(65.0-90.0)$ & $<0.001$ \\
\hline Lawton scale & 7.0 & $(6.0-8.0)$ & 3.0 & $(2.0-4.8)$ & $<0.001$ \\
\hline \multicolumn{6}{|l|}{ Diabetes characteristics } \\
\hline Duration (years) & 13.0 & $(7.0-19.0)$ & 13.0 & $(7.0-24.0)$ & 0.679 \\
\hline Cardiovascular disease & 547 & $(66.5 \%)$ & 25 & $(62.5 \%)$ & 0.729 \\
\hline Neuropathy & 75 & $(9.1 \%)$ & 7 & $(17.5 \%)$ & 0.092 \\
\hline Retinopathy & 27 & $(3.3 \%)$ & 0 & $(0 \%)$ & 0.631 \\
\hline Body mass index $\left(\mathrm{kg} / \mathrm{m}^{2}\right)$ & 24.8 & $(22.5-27.2)$ & 25.4 & $(21.9-29.3)$ & 0.336 \\
\hline Systolic blood pressure $(\mathrm{mmHg})$ & 130.0 & $(121.0-140.0)$ & 122.5 & $(|1| 2.3-135.8)$ & 0.019 \\
\hline Diastolic blood pressure $(\mathrm{mmHg})$ & 72.0 & $(64.0-79.0)$ & 74.5 & $(58.5-78.0)$ & 0.443 \\
\hline Blood hemoglobin Alc (\%) & 7.1 & $(6.5-7.9)$ & 7.1 & $(6.3-8.5)$ & 0.762 \\
\hline Fasting plasma glucose $(\mathrm{mg} / \mathrm{dL})$ & 130.0 & $(113.0-153.0)$ & 130.0 & $(104.3-157.8)$ & 0.755 \\
\hline Triglycerides (mg/dL) & 102.0 & $(73.0-147.0)$ & 140.0 & $(87.8-182.3)$ & 0.009 \\
\hline Low-density lipoprotein cholesterols $(\mathrm{mg} / \mathrm{dL})$ & 82.0 & $(68.0-99.0)$ & 78.0 & $(66.0-92.8)$ & 0.454 \\
\hline Blood creatinine $(\mathrm{mg} / \mathrm{dL})$ & 1.0 & $(0.8-1.3)$ & 1.2 & $(0.9-1.4)$ & 0.008 \\
\hline Estimated glomerular filtration rate $\left(\mathrm{mL} / \mathrm{min} / 1.73 \mathrm{~m}^{2}\right)$ & 71.3 & $(51.5-87.4)$ & 49.4 & $(39.0-65.1)$ & $<0.001$ \\
\hline Urine albumin-creatinine ratio $(\mathrm{mg} / \mathrm{g})$ & 24.5 & $(9.2-94.9)$ & 40.2 & $(14.1-108.8)$ & 0.063 \\
\hline Treatment & & & & & 0.445 \\
\hline Lifestyle modification & 84 & $(10.2 \%)$ & 3 & $(7.5 \%)$ & \\
\hline Oral medications only & 567 & $(68.9 \%)$ & 26 & $(65.0 \%)$ & \\
\hline Insulin only & 40 & $(4.9 \%)$ & I & $(2.5 \%)$ & \\
\hline Oral medications + insulin & 132 & $(16.0 \%)$ & 10 & $(25.0 \%)$ & \\
\hline
\end{tabular}

Note: ${ }^{\dagger} A D L<100$.

Abbreviation: $A D L$, activities of daily living.

Second, our study found that $18.5 \%$ of the patients who were evaluated through the MMSE were classified as exhibiting possible dementia, which was higher than the rate of $10 \%$ found in a community-based survey of older adults with diabetes. ${ }^{17}$ We speculate that the higher prevalence seen in this study was possibly due to the fact that cognition screening was conducted in a diabetes outpatient clinic, where patients may be more at risk or may exhibit more comorbid conditions. In fact, a previous survey reported a rate of possible dementia in $24.1 \%$ of patients in the outpatient department of a hospital in Taiwan, ${ }^{18}$ with rates of $12 \%$ being reported in the USA and
Brazil. $^{2,19}$ In our study, MMSE scores were negatively associated with glycemic control status according to A1C stratification defined as $<7.0 \%, 7.0-9.0 \%$, and $\geq 9.0 \%$. This association may be explained by a number of potential mechanisms, including insulin resistance/hyperinsulinemia, as well as the generation of advanced products of glycosylation in the nervous system. ${ }^{20}$ However, it is also possible that older patients with diabetes and concomitant cognitive dysfunction may be unable to follow complicated medication regimens and therefore their risk of hyperglycemia is increased. ${ }^{21}$ It is well known that older persons with diabetes are more likely to develop a series of 
Table 5 Characteristics of Participants with and without Dependence by IADL ${ }^{\dagger}$

\begin{tabular}{|c|c|c|c|c|c|}
\hline \multirow{3}{*}{$\begin{array}{l}\text { Demographic characteristics } \\
\text { Age (years) }\end{array}$} & \multicolumn{2}{|c|}{ IADL Independence } & \multicolumn{2}{|c|}{ IADL Dependence } & \multirow[t]{2}{*}{ p value } \\
\hline & & & & & \\
\hline & 70.0 & $(67.0-75.0)$ & 74.0 & $(68.0-80.0)$ & $<0.001$ \\
\hline Gender, n (\%) & & & & & $<0.001$ \\
\hline Female & 283 & $(72.0 \%)$ & 163 & $(34.7 \%)$ & \\
\hline Male & 110 & $(28.0 \%)$ & 307 & $(65.3 \%)$ & \\
\hline Educational level, n (\%) & & & & & 0.322 \\
\hline Illiterate & 47 & $(12.0 \%)$ & 68 & $(14.5 \%)$ & \\
\hline Literate & 345 & $(88.0 \%)$ & 400 & $(85.5 \%)$ & \\
\hline \multicolumn{6}{|l|}{ Geriatric characteristics } \\
\hline Polypharmacy & 226 & $(57.5 \%)$ & 286 & $(60.9 \%)$ & 0.354 \\
\hline Mini-mental state examination & 27.0 & $(25.0-29.0)$ & 26.0 & $(23.0-28.0)$ & $<0.001$ \\
\hline 5-item Geriatric Depression Scale & 0 & $(0-1.0)$ & 0 & $(0-1.0)$ & 0.120 \\
\hline Barthel index & 100.0 & $(100.0-100.0)$ & 100.0 & $(100.0-100.0)$ & $<0.001$ \\
\hline Lawton scale & 8.0 & $(8.0-8.0)$ & 6.0 & $(5.0-7.0)$ & $<0.001$ \\
\hline \multicolumn{6}{|l|}{ Diabetes characteristics } \\
\hline Duration (years) & 12.0 & $(7.0-18.0)$ & 14.0 & $(8.0-21.0)$ & 0.006 \\
\hline Cardiovascular disease & 251 & $(63.9 \%)$ & 321 & $(68.3 \%)$ & 0.194 \\
\hline Neuropathy & 31 & $(7.9 \%)$ & 51 & $(10.9 \%)$ & 0.173 \\
\hline Retinopathy & 12 & $(3.1 \%)$ & 15 & $(3.2 \%)$ & 1.000 \\
\hline Body mass index $\left(\mathrm{kg} / \mathrm{m}^{2}\right)$ & 24.6 & $(22.4-27.1)$ & 25.0 & $(22.6-27.4)$ & 0.337 \\
\hline Systolic blood pressure $(\mathrm{mmHg})$ & 130.0 & $(\mid 22.0-139.0)$ & 130.0 & $(\mid 20.0-140.0)$ & 0.528 \\
\hline Diastolic blood pressure $(\mathrm{mmHg})$ & 72.0 & $(65.0-79.0)$ & 71.0 & $(62.8-78.0)$ & 0.131 \\
\hline Blood hemoglobin Alc (\%) & 7.1 & $(6.5-7.8)$ & 7.1 & $(6.5-8.0)$ & 0.301 \\
\hline Fasting plasma glucose (mg/dL) & 128.0 & $(1 \mid 4.0-153.5)$ & 131.0 & $(111.0-152.3)$ & 0.908 \\
\hline Triglycerides (mg/dL) & 102.0 & $(70.0-145.0)$ & 105.0 & $(76.0-153.0)$ & 0.216 \\
\hline Low-density lipoprotein cholesterols $(\mathrm{mg} / \mathrm{dL})$ & 83.0 & $(69.0-99.0)$ & 82.0 & $(67.0-98.3)$ & 0.357 \\
\hline Blood creatinine $(\mathrm{mg} / \mathrm{dL})$ & 0.8 & $(0.7-I . I)$ & I.I & $(0.9-1.4)$ & $<0.001$ \\
\hline Estimated glomerular filtration rate $\left(\mathrm{mL} / \mathrm{min} / 1.73 \mathrm{~m}^{2}\right)$ & 76.7 & $(60.4 I-92.3)$ & 65.0 & $(44.8-80.9)$ & $<0.001$ \\
\hline Urine albumin-creatinine ratio $(\mathrm{mg} / \mathrm{g})$ & 19.6 & $(8.7-63.3)$ & 31.6 & $(10.4-143.4)$ & $<0.001$ \\
\hline Treatment & & & & & 0.005 \\
\hline Lifestyle modification & 40 & $(10.2 \%)$ & 47 & $(10.0 \%)$ & \\
\hline Oral medications only & 290 & $(73.8 \%)$ & 303 & $(64.5 \%)$ & \\
\hline Insulin only & 17 & $(4.3 \%)$ & 24 & $(5.1 \%)$ & \\
\hline Oral medications + insulin & 46 & (II.7\%) & 96 & $(20.4 \%)$ & \\
\hline
\end{tabular}

Note: ${ }^{\dagger}$ IADL $<8$.

Abbreviation: IADL, instrumental activities of daily living.

diabetic complications, which could affect the renal, neurological, and cardiovascular systems. Consequently, diabetic complications could cause physical and mental conditions to become even worse, leading to both physical and cognitive disability. ${ }^{22}$

Third, in this study, we also found that in addition to abnormalities in blood glucose control, renal dysfunction assessed by eGFR and UACR was associated with physical and cognitive impairment in diabetic patients, which is consistent with previous report. ${ }^{22,23}$ It has been proposed that chronic kidney disease (CKD) in older persons with diabetes may often coexist with risk factors for cognitive impairment, including hyperlipidemia, hypertension, and cardiovascular disease, which in turn can contribute to the development of dementia and physical disability. ${ }^{24,25}$ Pathophysiologically, CKD may result in vascular endothelial injury or renal insufficiency, both of which have been shown to exacerbate neuronal damage, resulting in alteration amyloid homeostasis. ${ }^{26}$ In addition, CKD can alter the homeostasis of phosphate calcium and vitamin $\mathrm{D}$, resulting in secondary hyperparathyroidism, which consequently contribute to muscle loss and bone fragility in CKD patients. ${ }^{27}$ In particular, our study found that older diabetic adults with cognitive and physical function became impaired even if their renal function just mildly 
Table 6 Logistic Regression Analysis for Predictors of MMSE, ADL, and IADL Impairment

\begin{tabular}{|c|c|c|c|c|c|c|c|}
\hline \multirow[t]{2}{*}{ MMSE Impairment } & \multicolumn{3}{|c|}{ Univariate } & \multicolumn{4}{|c|}{ Multivariate } \\
\hline & OR & $95 \% \mathrm{Cl}$ & $p$ value & OR & \multicolumn{2}{|c|}{$95 \% \mathrm{Cl}$} & p value \\
\hline Age (years) & 1.08 & $(I .06-I . I I)$ & $<0.001$ & 1.07 & \multicolumn{2}{|c|}{$(1.04-1.09)$} & $<0.001$ \\
\hline Diastolic blood pressure $(\mathrm{mmHg})$ & 0.98 & $(0.97-0.99)$ & 0.009 & 0.99 & \multicolumn{2}{|c|}{$(0.98-1.01)$} & 0.310 \\
\hline Cerebral vascular disease & 2.42 & $(1.29-4.54)$ & 0.006 & 2.43 & \multicolumn{2}{|c|}{$(1.25-4.74)$} & 0.009 \\
\hline Disease duration (years) & 1.03 & $(1.01-1.05)$ & 0.003 & 1.01 & \multicolumn{2}{|c|}{$(0.99-1.03)$} & 0.362 \\
\hline Blood hemoglobin Alc (\%) & 1.14 & $(1.01-1.29)$ & 0.034 & 1.10 & \multicolumn{2}{|c|}{$(0.94-1.28)$} & 0.226 \\
\hline Fasting plasma glucose (mg/dL) & 1.00 & $(1.00-1.01)$ & 0.024 & 1.00 & \multicolumn{2}{|c|}{$(1.00-1.01)$} & 0.257 \\
\hline Blood creatinine (mg/dL) & 1.35 & $(1.07-1.70)$ & 0.011 & 0.75 & \multicolumn{2}{|c|}{$(0.45-1.25)$} & 0.272 \\
\hline Estimated glomerular filtration rate $\left(\mathrm{mL} / \mathrm{min} / 1.73 \mathrm{~m}^{2}\right)$ & 0.98 & $(0.98-0.99)$ & $<0.001$ & 0.99 & \multicolumn{2}{|c|}{$(0.97-1.00)$} & 0.026 \\
\hline 5-item Geriatric Depression Scale & 1.35 & $(1.10-1.66)$ & 0.005 & - & - & - & - \\
\hline Barthel index & 0.95 & $(0.93-0.98)$ & $<0.001$ & - & - & - & - \\
\hline Lawton scale & 0.69 & $(0.62-0.75)$ & $<0.001$ & - & - & - & - \\
\hline \multirow[t]{2}{*}{ ADL Impairment } & \multicolumn{3}{|c|}{ Univariate } & \multicolumn{4}{|c|}{ Multivariate } \\
\hline & OR & $95 \% \mathrm{Cl}$ & p value & OR & \multicolumn{2}{|c|}{$95 \% \mathrm{Cl}$} & p value \\
\hline Age (years) & 1.12 & $(1.08-1.17)$ & $<0.001$ & 1.13 & \multirow{4}{*}{\multicolumn{2}{|c|}{$\begin{array}{l}(1.08-1.19) \\
(0.11-0.53) \\
(0.97-1.00) \\
(0.97-1.00)\end{array}$}} & $<0.001$ \\
\hline Male vs Female & 0.39 & $(0.19-0.79)$ & 0.009 & 0.24 & & & $<0.001$ \\
\hline Systolic blood pressure $(\mathrm{mmHg})$ & 0.98 & $(0.97-1.00)$ & 0.017 & 0.98 & & & 0.013 \\
\hline Estimated glomerular filtration rate $\left(\mathrm{mL} / \mathrm{min} / 1.73 \mathrm{~m}^{2}\right)$ & 0.98 & $(0.96-0.99)$ & $<0.001$ & 0.98 & & & 0.044 \\
\hline Mini-mental state examination & 0.79 & $(0.73-0.84)$ & $<0.001$ & - & - & - & - \\
\hline 5-item Geriatric Depression Scale & 1.79 & $(1.32-2.42)$ & $<0.001$ & - & - & - & - \\
\hline Lawton scale & 0.43 & $(0.36-0.52)$ & $<0.001$ & - & - & - & - \\
\hline \multirow[t]{2}{*}{ IADL Impairment } & \multicolumn{3}{|c|}{ Univariate } & \multicolumn{4}{|c|}{ Multivariate } \\
\hline & OR & $95 \% \mathrm{Cl}$ & p value & OR & \multicolumn{2}{|c|}{$95 \% \mathrm{Cl}$} & p value \\
\hline Age (years) & 1.07 & $(1.05-1.10)$ & $<0.001$ & 1.07 & \multicolumn{2}{|c|}{$(1.04-1.10)$} & $<0.001$ \\
\hline Male vs Female & 4.85 & $(3.62-6.48)$ & $<0.001$ & 4.91 & \multicolumn{2}{|c|}{$(3.49-6.90)$} & $<0.001$ \\
\hline Disease duration (years) & 1.02 & $(1.01-1.04)$ & 0.005 & 1.01 & \multicolumn{2}{|c|}{$(0.99-1.03)$} & 0.233 \\
\hline Oral medications only vs Diet & 0.89 & $(0.57-1.40)$ & 0.610 & 1.19 & \multicolumn{2}{|c|}{$(0.72-1.98)$} & 0.501 \\
\hline Insulin only vs Diet & 1.20 & $(0.57-2.55)$ & 0.632 & 0.90 & \multicolumn{2}{|c|}{$(0.38-2.13)$} & 0.812 \\
\hline Oral medications + insulin vs Diet & 1.78 & $(1.03-3.08)$ & 0.040 & 2.32 & \multicolumn{2}{|c|}{$(1.24-4.34)$} & 0.009 \\
\hline Blood creatinine (mg/dL) & 3.24 & $(2.31-4.54)$ & $<0.001$ & 1.21 & \multicolumn{2}{|c|}{$(0.73-2.01)$} & 0.458 \\
\hline Estimated glomerular filtration rate $\left(\mathrm{mL} / \mathrm{min} / 1.73 \mathrm{~m}^{2}\right)$ & 0.98 & $(0.98-0.99)$ & $<0.001$ & 0.99 & $(0.9$ & $1.00)$ & 0.219 \\
\hline Mini-mental state examination & 0.89 & $(0.86-0.93)$ & $<0.001$ & - & - & - & - \\
\hline Barthel index & 0.88 & $(0.81-0.96)$ & 0.003 & - & - & - & - \\
\hline
\end{tabular}

Abbreviations: MMSE, mini-mental state examination; ADL, activities of daily living; IADL, instrumental activities of daily living.

decreased (eg, a mean eGFR of around $60 \mathrm{~mL} / \mathrm{min}$ ), which was in line with two previous studies. ${ }^{28,29}$ It has been suggested that the association of CKD with cognition disorder and functional disability may date back to the early CKD stages. Of note, this study did not show an association between the other diabetic microvascular complications (eg, neuropathy and retinopathy) and cognitive and physical function impairment. Some mechanisms have already been proposed to link microvascular complications to the pathogenesis of cognitive impairment in diabetes, including oxidative stress, inflammation, and others. ${ }^{30}$ However, although these may be considered common mechanisms for neuropathy, retinopathy and cognitive impairment, there may be other variables that could be of great relevance for the association of neuropathy and retinopathy and physical and cognitive impairment in specific patients. ${ }^{31,32}$ Further studies are necessary to clarify this issue. 
Fourth, in our study patients, $4.6 \%$ experienced at least one physical functional problem assessed by ADL with a score less than or equal to 60 seen in $0.8 \%$. Furthermore, $470(54.5 \%)$ patients exhibited at least one abnormal IADL component. This prevalence of functional limitation was similar to that which occurred in a previous study performed in a community of older persons with diabetes. ${ }^{33}$ It has been proposed that disabilities due to diabetes may be caused by associated complications, ${ }^{34}$ as well as other prevalent comorbidities in adults with diabetes. ${ }^{35}$ As shown in our patients, systolic blood pressure and eGFR were positively correlated with ADL abnormality. However, these disabilities in diabetes can be treated and minimized (eg, use of physical therapy for deconditioning and gait training) through early screening of physical function in older persons.

In our study, it was shown that older diabetic adults with cognitive or physical impairment have lower diastolic or systolic blood pressure in comparison with those with normal scores. Hypertension is an important risk factor for vascular disease patients, as it may lead to cognitive impairment due to stroke, and therefore greater disability in the older people. ${ }^{36,37}$ However, in older people, inappropriate low blood pressure can also increase the risk of dementia, as well as cause physical impairment. ${ }^{38,39}$ It has been proposed that low blood pressure can cause hypoperfusion in vital organs and tissues, including the brain, heart and musculoskeletal systems, thus resulting in diminished cognitive and physical performance. ${ }^{40,41}$ Overall, it is suggested that blood pressure management be personalized for the prevention of cognitive and physical function impairment in older persons with diabetes.

In our reports, $8.3 \%$ of the patients were diagnosed as having symptoms of depression, which in turn were associated with cognitive and physical abnormality (measured by MMSE and ADL scores). It remains well known that a depressive state is an important factor affecting cognitive dysfunction, ${ }^{42}$ functional limitations, ${ }^{43}$ and disease management and the quality of life in older populations with chronic diseases. ${ }^{44,45}$ In older persons with diabetes, lower cognition or emotional well-being may decrease their capability to selfmanage their disease; therefore, early detection of cognitive impairment, depressive symptoms, and physical disability in individuals with diabetes is particularly important so that supporting resources can be arranged.

Similar to other studies, ${ }^{35,46}$ we found that patients with a longer duration of diabetes and older age had lower cognitive and physical function test scores. It has been proposed that mechanisms relating age and diabetes duration to dementia and disability may be mediated by chronic low-level inflammation, which is associated with hyperinsulinaemia and/or insulin resistance, advanced glycation end-products, atherosclerosis and other vascular complications. ${ }^{1,3}$ In addition, this study also observed that the proportion of participants who used insulin and/ or oral glucose-lowering drugs was higher in patients with cognitive and physical function impairment. Because there are no studies showing that glucose-lowering intervention or glucose lowering medications ${ }^{47-49}$ were efficacious to modulating the risk of cognitive and physical decline, we believe that the correlation was possibly indirect due to a longer duration and severity of the underlying disease.

In order to achieve control goals, diabetes treatment regimens have become more complex, resulting in older persons with diabetes with concomitant disabilities possibly being unable to follow complicated regimens. ${ }^{2,50}$ Moreover, previous studies have found limitations in ADL, and cognitive impairment could increase the risk of fall, thus resulting in fractures, cerebral trauma, morbidities, mortality, and higher health-care costs. ${ }^{51}$ Particularly, during the outbreak of Severe Acute Respiratory Syndrome Coronavirus 2 (SARS-CoV-2) infection and resulting coronavirus disease 2019, physical dysfunction in older persons with diabetes may have been exacerbated due to home confinement causing a reduction in physical activity, an increase in sedentary behavior and unhealthy meal patterns, all of which further bring deleterious effects on diabetes control. ${ }^{52,53}$ In these circumstances, through assessments of cognition and physical function in older persons with diabetes, individualization and simplification of treatment plans can be implemented. Overall, our study findings may be of value to physicians, as the use of assessment could help to detect decline physical and cognitive functions in older persons with diabetes, in turn prompting earlier intervention.

The study had certain limitations. First, our subjects included those who were capable of visiting our outpatient department, and as a result, they may have had better physical function causing the prevalence of disability to possibly have been underestimated. Second, MMSE scores are influenced by several factors such as age, educational level, and premorbid intelligence of the patient, ${ }^{8}$ and may therefore not detect mild cognitive impairment. Third, we did not evaluate the personal history of lifestyle habits, such as exercise, smoking habits and alcohol consumption. These factors may have a significant impact on cognitive 
function. Fourth, our findings were based upon data from a single integrated health system and may not be generalizable for other populations. Besides, multiple comparisons were done in the statistical analysis, which may lead to draw false-positive conclusion. ${ }^{11,12}$ However, this study was exploratory in characteristics to examine potential risk factors associated with physical and cognitive dysfunction in older diabetic patients, and thus no correction for multiple comparisons was performed. ${ }^{11,12}$ Nevertheless, additional dedicated studies are needed to confirm the results. Fifth, although we found the relationship between blood pressure and cognitive and physical problems, we did not further analyze classes of antihypertensive medication. According to a recently published meta-analysis, it was reported that there was no evidence that a specific antihypertensive medication class was more effective than others in lowering risk of dementia. ${ }^{54}$ Lastly, the study employed a cross-sectional design that did not include a control population of older adults without diabetes. Thus, a causal relationship between diabetes-associated factors, and cognitive and physical dysfunction could not be established. Further research remains necessary in order to establish a more definite conclusion.

\section{Conclusion}

In summary, this study by designing physical and cognitive function assessment service found that both cognitive and physical function impairment were common in older persons with type 2 diabetes at an outpatient clinic. Age, glycemic control, blood pressure levels and renal function were all associated with cognitive dysfunction and physical limitations. We recommend an integration of cognitive and physical function assessment into clinical practice in older individuals with diabetes, particularly those with risk factors, in order to expedite provision of optimized management plans.

\section{Institutional Review Board Statement}

The study was conducted according to the guidelines of the Declaration of Helsinki, and approved by the Institutional Review Board of Taichung Veterans General Hospital (protocol code CE18117A).

\section{Informed Consent Statement}

Patient consent was waived by the Institutional Review Board of Taichung Veterans General Hospital due to the retrospective nature of this study.

\section{Acknowledgments}

The authors are grateful to the Biostatistics Task Force of Taichung Veterans General Hospital for assistance with statistical analysis in this study.

\section{Funding}

This work was supported by grants from Taichung Veterans General Hospital, Taiwan (TCVGH-1108201E) and Rong Sing Medical Foundation (RSMF-1090163).

\section{Disclosure}

The authors report no conflicts of interest in this work.

\section{References}

1. Biessels GJ, Despa F. Cognitive decline and dementia in diabetes mellitus: mechanisms and clinical implications. Nat Rev Endocrinol. 2018;14(10):591-604. doi:10.1038/s41574-018-0048-7

2. Munshi M, Grande L, Hayes M, et al. Cognitive dysfunction is associated with poor diabetes control in older adults. Diabetes Care. 2006;29(8):1794-1799. doi:10.2337/dc06-0506

3. Wong E, Backholer K, Gearon E, et al. Diabetes and risk of physical disability in adults: a systematic review and meta-analysis. Lancet Diabetes Endocrinol. 2013;1(2):106-114. doi:10.1016/s22138587(13)70046-9

4. Li CL, Chiu YC, Bai YB, Lin JD, Stanaway F, Chang HY. The co-occurrence of depressive symptoms and cognitive impairment and its relationship with self-care behaviors among community dwelling older adults with diabetes. Diabetes Res Clin Pract. 2017;129:73-78. doi:10.1016/j.diabres.2017.03.025

5. Li CL, Chiu YC, Chang HY, Hsu KH, Bai YB, Wang HH. Association of geriatric conditions and cardiovascular diseases with disability in older adults with diabetes: findings from a nationally representative survey. Geriatr Gerontol Int. 2013;13(3):563-570. doi:10.1111/j.1447-0594.2012.00935.x

6. Liu HY, Tsai WC, Chiu MJ, Tang LY, Lee HJ, Shyu YL. Mild cognitive impairment in combination with comorbid diabetes mellitus and hypertension is negatively associated with health-related quality of life among older persons in Taiwan. Qual Life Res. 2019;28 (5):1281-1291. doi:10.1007/s11136-019-02101-3

7. Lee IT, Hsu CC, Sheu WH, Su SL, Wu YL, Lin SY. Pay-forperformance for shared care of diabetes in Taiwan. $J$ Formos Med Assoc. 2019;118(Suppl 2):S122-s129. doi:10.1016/j. jfma.2019.08.011

8. Wu JJ, Weng SC, Liang CK, et al. Effects of kidney function, serum albumin and hemoglobin on dementia severity in the oldest old people with newly diagnosed Alzheimer's disease in a residential aged care facility: a cross-sectional study. BMC Geriatr. 2020;20 (1):391. doi:10.1186/s12877-020-01789-0

9. Chen TB, Yiao SY, Sun Y, et al. Comorbidity and dementia: a nationwide survey in Taiwan. PLoS One. 2017;12(4):e0175475. doi:10.1371/journal.pone. 0175475

10. Hoyl MT, Alessi CA, Harker JO, et al. Development and testing of a five-item version of the Geriatric Depression Scale. J Am Geriatr Soc. 1999;47(7):873-878. doi:10.1111/j.1532-5415.1999.tb03848.x

11. Althouse AD. Adjust for multiple comparisons? It's not that simple. Ann Thorac Surg. 2016;101(5):1644-1645. doi:10.1016/j. athoracsur.2015.11.024

12. Bender R, Lange S. Adjusting for multiple testing - when and how? $J$ Clin Epidemiol. 2001;54(4):343-349. doi:10.1016/s0895-4356(00) 00314-0 
13. Sinclair AJ, Girling AJ, Bayer AJ. Cognitive dysfunction in older subjects with diabetes mellitus: impact on diabetes self-management and use of care services. All Wales Research into Elderly (AWARE) Study. Diabetes Res Clin Pract. 2000;50(3):203-212. doi:10.1016/ s0168-8227(00)00195-9

14. Nixon AC, Brown J, Brotherton A, et al. Implementation of a frailty screening programme and Geriatric Assessment Service in a nephrology centre: a quality improvement project. $J$ Nephrol. 2021;34(4):1215-1224. doi:10.1007/s40620-020-00878-y

15. Presley CJ, Krok-Schoen JL, Wall SA, et al. Implementing a multidisciplinary approach for older adults with cancer: geriatric oncology in practice. BMC Geriatr. 2020;20(1):231. doi:10.1186/ s12877-020-01625-5

16. American Diabetes Association 4. Comprehensive medical evaluation and assessment of comorbidities: standards of medical care in diabetes-2021. Diabetes Care. 2021;44(Supp11):S40-s52. doi:10.2337/dc21-S004.

17. Xiu S, Zheng Z, Liao Q, Chan P. Different risk factors for cognitive impairment among community-dwelling elderly, with impaired fasting glucose or diabetes. Diabetes Metab Syndr Obes. 2019;12:121-130. doi:10.2147/dmso.S180781

18. Tai SY, Huang SW, Hsu CL, Yang CH, Chou MC, Yang YH. Screening dementia in the outpatient department: patients at risk for dementia. ScientificWorldJournal. 2014;2014:138786. doi:10.1155/ 2014/138786

19. Alencar RC, Cobas RA, Gomes MB. Assessment of cognitive status in patients with type 2 diabetes through the Mini-Mental Status Examination: a cross-sectional study. Diabetol Metab Syndr. 2010;2:10. doi:10.1186/1758-5996-2-10

20. Karan NS. Assessment of the cognitive status in diabetes mellitus. J Clin Diagn Res. 2012;6(10):1658-1662. doi:10.7860/jcdr/2012/ 4837.2649

21. Thorpe CT, Gellad WF, Good CB, et al. Tight glycemic control and use of hypoglycemic medications in older veterans with type 2 diabetes and comorbid dementia. Diabetes Care. 2015;38 (4):588-595. doi:10.2337/dc14-0599

22. Hong X, Chen X, Chu J, et al. Multiple diabetic complications, as well as impaired physical and mental function, are associated with declining balance function in older persons with diabetes mellitus. Clin Interv Aging. 2017;12:189-195. doi:10.2147/cia.S123985

23. Umegaki H, Iimuro S, Shinozaki T, et al. Risk factors associated with cognitive decline in the elderly with type 2 diabetes: pooled logistic analysis of a 6-year observation in the Japanese Elderly Diabetes Intervention Trial. Geriatr Gerontol Int. 2012;12 Suppl 1:110-116. doi:10.1111/j.1447-0594.2011.00818.x

24. Major RW, Oozeerally I, Dawson S, Riddleston H, Gray LJ, Brunskill NJ. Aspirin and cardiovascular primary prevention in non-end stage chronic kidney disease: a meta-analysis. Atherosclerosis. 2016;251:177-182. doi:10.1016/j.atherosclerosis.2016.06.013

25. Jin SH, Park YS, Park YH, Chang HJ, Kim SR. Comparison of gait speed and peripheral nerve function between chronic kidney disease patients with and without diabetes. Ann Rehabil Med. 2017;41 (1):72-79. doi:10.5535/arm.2017.41.1.72

26. Bugnicourt JM, Godefroy O, Chillon JM, Choukroun G, Massy ZA. Cognitive disorders and dementia in CKD: the neglected kidney-brain axis. $J$ Am Soc Nephrol. 2013;24(3):353-363. doi:10.1681/asn.2012050536

27. Molina P, Carrero JJ, Bover J, et al. A modulator of musculoskeletal health in chronic kidney disease. $J$ Cachexia Sarcopenia Muscle. 2017;8(5):686-701. doi:10.1002/jcsm.12218

28. Viscogliosi G, De Nicola L, Vanuzzo D, Giampaoli S, Palmieri L, Donfrancesco C. Mild to moderate chronic kidney disease and functional disability in community-dwelling older adults. The Cardiovascular risk profile in Renal patients of the Italian Health Examination Survey (CARHES) study. Arch Gerontol Geriatr. 2019;80:46-52. doi:10.1016/j.archger.2018.10.001
29. Kong JY, Kim JS, Kang MH, Hwang HS, Won CW, Jeong KH. Renal dysfunction is associated with decline of cognitive function in community-dwelling older adults: Korean frailty and aging cohort study. BMC Geriatr. 2020;20(1):462. doi:10.1186/s12877-02001862-8

30. Umegaki H, Kawamura T, Umemura T, Kawano N. Factors associated with cognitive decline in older adults with type 2 diabetes mellitus during a 6-year observation. Geriatr Gerontol Int. 2015;15 (3):302-310. doi:10.1111/ggi.12273

31. Kawamura T, Umemura T, Hotta N. Curious relationship between cognitive impairment and diabetic retinopathy. J Diabetes Investig. 2015;6(1):21-23. doi:10.1111/jdi.12234

32. Moreira RO, Soldera AL, Cury B, Meireles C, Kupfer R. Is cognitive impairment associated with the presence and severity of peripheral neuropathy in patients with type 2 diabetes mellitus? Diabetol Metab Syndr. 2015;7:51. doi:10.1186/s13098-015-0045-0

33. Yang YC, Lin MH, Wang CS, et al. Geriatric syndromes and quality of life in older adults with diabetes. Geriatr Gerontol Int. 2019;19 (6):518-524. doi:10.1111/ggi.13654

34. Bruce DG, Davis WA, Davis TM. Longitudinal predictors of reduced mobility and physical disability in patients with type 2 diabetes: the Fremantle Diabetes Study. Diabetes Care. 2005;28(10):2441-2447. doi:10.2337/diacare.28.10.2441

35. Kalyani RR, Saudek CD, Brancati FL, Selvin E. Association of diabetes, comorbidities, and $\mathrm{A} 1 \mathrm{C}$ with functional disability in older adults: results from the National Health and Nutrition Examination Survey (NHANES), 1999-2006. Diabetes Care. 2010;33 (5):1055-1060. doi:10.2337/dc09-1597

36. Stuck AE, Walthert JM, Nikolaus T, Büla CJ, Hohmann C, Beck JC. Risk factors for functional status decline in community-living elderly people: a systematic literature review. Soc Sci Med. 1999;48 (4):445-469. doi:10.1016/s0277-9536(98)00370-0

37. Abete P, Della-Morte D, Gargiulo G, et al. Cognitive impairment and cardiovascular diseases in the elderly. A heart-brain continuum hypothesis. Ageing Res Rev. 2014;18:41-52. doi:10.1016/j. arr.2014.07.003

38. Guo Z, Viitanen M, Winblad B. Clinical correlates of low blood pressure in very old people: the importance of cognitive impairment. J Am Geriatr Soc. 1997;45(6):701-705. doi:10.1111/ j.1532-5415.1997.tb01473.x

39. Paran E, Anson O, Reuveni H. Blood pressure and cognitive functioning among independent elderly. Am J Hypertens. 2003;16 (10):818-826. doi:10.1016/s0895-7061(03)01005-7

40. Duschek S, Schandry R. Reduced brain perfusion and cognitive performance due to constitutional hypotension. Clin Auton Res. 2007;17(2):69-76. doi:10.1007/s10286-006-0379-7

41. Ogliari G, Westendorp RG, Muller M, et al. Blood pressure and 10-year mortality risk in the Milan Geriatrics $75+$ cohort study: role of functional and cognitive status. Age Ageing. 2015;44(6):932-937. doi:10.1093/ageing/afv141

42. Richard E, Reitz C, Honig LH, et al. Late-life depression, mild cognitive impairment, and dementia. JAMA Neurol. 2013;70 (3):374-382. doi:10.1001/jamaneurol.2013.603

43. Song HJ, Meade K, Akobundu U, Sahyoun NR. Depression as a correlate of functional status of community-dwelling older adults: utilizing a short-version of 5-item Geriatric Depression Scale as a screening tool. J Nutr Health Aging. 2014;18(8):765-770. doi:10.1007/s12603-014-0542-0

44. Jiménez-Cebrián AM, Becerro-de-bengoa-vallejo R, Losa-Iglesias ME, et al. The impact of depression symptoms in patients with Parkinson's Disease: a novel case-control investigation. Int $J$ Environ Res Public Health. 2021;18(5). doi:10.3390/ ijerph 18052369

45. Gold SM, Köhler-Forsberg O, Moss-Morris R, et al. Comorbid depression in medical diseases. Nat Rev Dis Primers. 2020;6(1):69. doi:10.1038/s41572-020-0200-2 
46. Bruce DG, Davis WA, Casey GP, et al. Predictors of cognitive impairment and dementia in older people with diabetes. Diabetologia. 2008;51(2):241-248. doi:10.1007/s00125-007-0894-7

47. Fink HA, Jutkowitz E, McCarten JR, et al. Pharmacologic interventions to prevent cognitive decline, mild cognitive impairment, and clinical Alzheimer-type dementia: a systematic review. Ann Intern Med. 2018;168(1):39-51. doi:10.7326/m17-1529

48. Areosa Sastre A, Vernooij RW, González-Colaço Harmand M, Martínez G. Effect of the treatment of Type 2 diabetes mellitus on the development of cognitive impairment and dementia. Cochrane Database Syst Rev. 2017;6(6):Cd003804. doi:10.1002/14651858. CD003804.pub2

49. Yau CK, Eng C, Cenzer IS, Boscardin WJ, Rice-Trumble K, Lee SJ. Glycosylated hemoglobin and functional decline in community-dwelling nursing home-eligible elderly adults with diabetes mellitus. J Am Geriatr Soc. 2012;60(7):1215-1221. doi:10.1111/j.1532-5415.2012.04041.x

50. Shang Y, Fratiglioni L, Vetrano DL, Dove A, Welmer AK, Xu W. Not only diabetes but also prediabetes leads to functional decline and disability in older adults. Diabetes Care. 2021;44(3):690-698. doi: $10.2337 / \mathrm{dc} 20-2232$
51. Pijpers E, Ferreira I, de Jongh RT, et al. Older individuals with diabetes have an increased risk of recurrent falls: analysis of potential mediating factors: the Longitudinal Ageing Study Amsterdam. Age Ageing. 2012;41(3):358-365. doi:10.1093/ageing/afr145

52. Marçal IR, Fernandes B, Viana AA, Ciolac EG. The urgent need for recommending physical activity for the management of diabetes during and beyond covid-19 outbreak. Front Endocrinol (Lausanne). 2020;11:584642. doi:10.3389/fendo.2020.584642

53. Trevissón-Redondo B, López-López D, Pérez-Boal E, et al. Use of the Barthel index to assess activities of daily living before and after SARS-COVID 19 infection of Institutionalized Nursing home patients. Int $J$ Environ Res Public Health. 2021;18(14). doi:10.3390/ijerph18147258

54. Ding J, Davis-Plourde KL, Sedaghat S, et al. Antihypertensive medications and risk for incident dementia and Alzheimer's disease: a meta-analysis of individual participant data from prospective cohort studies. Lancet Neurol. 2020;19(1):61-70. doi:10.1016/s14744422(19)30393-x

\section{Publish your work in this journal}

Diabetes, Metabolic Syndrome and Obesity: Targets and Therapy is an international, peer-reviewed open-access journal committed to the rapid publication of the latest laboratory and clinical findings in the fields of diabetes, metabolic syndrome and obesity research. Original research, review, case reports, hypothesis formation, expert opinion and commentaries are all considered for publication. The manuscript management system is completely online and includes a very quick and fair peer-review system, which is all easy to use. Visit http://www.dovepress.com/testimonials.php to read real quotes from published authors. 